\title{
Lineamientos estratégicos para la consolidación de una escuela inteligente y su noción de felicidad
}

\author{
Aranguren Peraza, Gilberto Natividad \\ Lineamientos estratégicos para la consolidación de una escuela inteligente y su noción de felicidad \\ Revista Educación, vol. 44, núm. 2, 2020 \\ Universidad de Costa Rica, Costa Rica \\ Disponible en: http://www.redalyc.org/articulo.oa?id=44062184006 \\ DOI: https://doi.org/10.15517/revedu.v44i2.37605
}

Esta obra está bajo una Licencia Creative Commons Atribución-NoComercial-SinDerivar 3.0 Internacional. 


\title{
Lineamientos estratégicos para la consolidación de una escuela inteligente y su noción
} de felicidad

\author{
Gilberto Natividad Aranguren Peraza \\ Universidad Pedagógica Experimental Libertador \\ (UPEL), Venezuela \\ gilberap@gmail.com \\ iD http://orcid.org/0000-0003-1480-9401
}

Strategic Guidelines for Consolidating Smart Schools and the Notion of Happiness

DOI: https://doi.org/10.15517/revedu.v44i2.37605

Redalyc: http://www.redalyc.org/articulo.oa?id=44062184006

Recepción: 01 Agosto 2019

Aprobación: 29 Febrero 2020

\section{Resumen:}

El ensayo describe la trascendencia de la escuela inteligente y su incidencia en la formación de los estados mentales para ser feliz. La escuela tiene como responsabilidad crear espacios sociales de índole creativa, para ello es importante hacer esfuerzos con el personal docente e investigador, así como desarrollar procesos para que el estudiantado tome distancia de los hábitos inadecuados aportados por generaciones pasadas. La escuela debe contribuir en varios aspectos de la vida de la persona como la construcción del sentido común, desarrollar competencias cognitivas y sociales, fortalecer interacciones mediante estrategias cooperativas, de campo y recreativas, generar reflexiones y sistematizaciones de la práctica, hacer esfuerzos en la reconstrucción de las relaciones dadas entre el personal docente y estudiantado, vincular la institución con agentes externos, valorar el trabajo y democratizar el poder escolar. Estas propuestas se hacen para contribuir a que las instituciones educativas se hagan más inteligentes. Una conclusión de interés es señalar que las escuelas orientadas mediante rígidas estructuras ya sean en la administración de la enseñanza o en las metodologías utilizadas para ese fin, poco favorecen estados mentales flexibles facilitadores de la creatividad y del aprendizaje.

Palabras Clave: Escuela Inteligente, Felicidad, Estados Mentales.

\section{ABstract:}

The essay describes the transcendence of Smart Schools and their impact on developing mental states of happiness. Schools are responsible for building social and creative surroundings through faculty and researchers and develop approaches so students can distance themselves from any inappropriate habits from past generations. Schools should also help contribute to various aspects of student life such as: help develop common sense as well as cognitive and social skills, strengthen interactions through cooperative learning and recreational strategies, develop thought and systematize drills, strive to rebuild teacher-student relationships, connect their schools with external agents, appreciate the value of work and democratize school authority. These efforts all contribute to making schools smarter. It is important to state that schools that operate according to rigid structures either regarding their administration or teaching methodologies do not foster mental states among students that promote creativity and learning.

KEYWORDS: Smart Schools, Happiness, State of Mind.

\section{INTRODUCCIÓN}

Hay una gran necesidad de hacer de la escuela una institución inteligente, esto supone una organización capaz de entenderse a sí misma como un escenario de interacción social y humana. La escuela es una institución donde ocurren innumerables relaciones, por ello el aprendizaje surge como un producto de dichas interacciones. La noción de una escuela inteligente nace de la idea de una organización abierta al aprendizaje y favorecedora del bienestar de cada uno de sus miembros.

La escuela debe mirarse como un sistema con vida, tal como lo plantea Capra (2000), cuando señala que las "sociedades humanas son por tanto tipos muy distintos de sistemas vivos" (p. 222), dada su diferencia con organismos que poseen funciones ya determinadas, porque la escuela, al igual que otras organizaciones 
sociales, interactúa de un modo diferente con el ambiente y sus funciones solo son reguladas, ya sea por sistemas externos o por las personas que la constituyen.

Por ser una institución organizada, por una parte, con estatutos y reglamentos, pero, por otra, por las interacciones dadas en su seno, la escuela tiene como meta favorecer espacios para el bienestar:

Aquí nos referimos a otro tipo de bienestar. La diversidad de enfoques sobre el bienestar justifica la conveniencia de 'poner un apellido' para concretar de qué bienestar hablamos: bienestar material, bienestar social, bienestar físico, bienestar profesional (engagement), bienestar emocional, bienestar subjetivo, bienestar psicológico, bienestar hedónico, bienestar eudemónico. Todos estos tipos de bienestar deberían formar parte de la educación, ya que nos orientan en el camino a seguir (Bisquerra y Hernández, 2017, p. 58).

Por esto, es imprescindible considerar que el bienestar posee una dimensión personal y colectiva, esto supone tener en cuenta la trascendencia de la persona en su desarrollo integral dentro de las organizaciones escolares, para alcanzar metas que beneficien el logro de la felicidad, tanto individual como en su trascendencia social.

La escuela requiere ser entendida desde un marco el cual desestructure las nociones o teorías basadas en enfoques psicométricos que han consolidado conceptos tales como el coeficiente intelectual (García, 2016), lejos de la comprensión de que el mundo es complejo y dinámico, y de que existen otras propuestas que consideran, por ejemplo, el desarrollo de las competencias emocionales favorecedoras de la consolidación de aulas felices.

Las sociedades y las escuelas acordaron favorecer una noción de inteligencia basada exclusivamente en la cognición, sin considerar propuestas con base en el bienestar emocional de las personas que intervienen en el proceso educativo (Prieto, 2018 y Bisquerra y Hernández, 2017). La reforma del pensamiento reconsidera los modos de ver el mundo con diversas inteligencias (Fernández, 2013), siendo necesario que la escuela pase del determinismo y absolutismo a una estructura dinámica y talentosa para los tiempos actuales, "el problema se reduce a lo siguiente: no aplicamos nuestros conocimientos. En las escuelas, los maestros enseñan y los alumnos aprenden como hace veinte o cincuenta años" (Perkins, 1999, p. 16), hoy las prácticas escolares siguen siendo las mismas en la mayoría de las instituciones educativas, aunque existen esfuerzos en considerar las tecnologías de la información como herramientas en la enseñanza, creándose nuevos modelos de ambientes pedagógicos con una gran influencia en los jóvenes, evidenciándose el uso de la Internet como un medio de transmisión y manipulación de la información, así como en la construcción del conocimiento (Carro y Lima, 2017); pero, también, existe la necesidad de favorecer espacios sociales creativos basados en las emociones y en el desarrollo de la inteligencia social.

La escuela, entendida como una institución social, organizada y establecida con una función especializada, cuenta con un contexto propio y un conjunto de personas responsables del cumplimiento de una serie de tareas específicas y reguladas por distintos sistemas (Fernández, 2001), enfrenta con valentía adversidades de tipo social, económica, cultural y política, por lo que su práctica no puede seguir siendo la de siempre. Las deficiencias que tiene no facilitan responder con nociones avanzadas en prácticas y construcciones teóricas de gran alcance, siendo imprescindible pensar en una escuela orgánica, pensante y humanamente inteligente. Es decir, una institución que haga uso del conocimiento, la información, la reflexión y del sentido común, para el desarrollo y fortalecimiento de los estados mentales de todas las personas que la constituyen.

La escuela inteligente mira con detenimiento al estudiantado en sus necesidades y dificultades, en la evaluación, en el gobierno escolar, la organización y estructuración, y en la formación docente, como categorías de análisis y revisión constantes durante el ejercicio de su práctica (Aranguren, 2017). Para ello, el aprendizaje reflexivo es el camino metodológico para la consolidación y profundización de los cambios internos.

Es importante comprender que la infelicidad se ha convertido en un problema de gran trascendencia humana y social, conduciendo a la persona a vivir y a experimentar situaciones y condiciones que la hacen sentirse insatisfecha. La escuela inteligente debe brindar herramientas a su personal docente y al estudiantado 
para que superen, mediante claras nociones de destinos, aquellos procesos adversos a su crecimiento personal y social.

Considerando lo anterior, el ensayo tiene como propósito brindar una serie de lineamientos estratégicos que permitan la comprensión de la escuela inteligente en el marco de la consolidación de una noción de felicidad como aspiración vivencial - humana de todas las personas que la integran.

\section{LA ESCUELA INTELIGENTE}

Una escuela inteligente es aquella institución humana promotora del desarrollo del pensamiento, del cultivo del espíritu y de las emociones. Siendo necesario un nuevo modo conceptual de la escuela, donde las percepciones, emociones y los comportamientos son estructuras vitales para la comprensión de los fenómenos humanos, científicos, culturales y naturales. Busca mejorar y atender a estudiantes con dificultades de cualquier índole y en riesgo social, evaluar con nuevos criterios, darle sentido a la administración escolar, reestructurar permanentemente los procesos y la formación del personal docente desde los mismos centros educativos. La tendencia integracionista de la escuela inteligente busca que la persona no se fragmente, y comprenda lo fraccionado de la sociedad para unirlo. De manera, que la escuela deberá actuar en contraposición a esta lógica, dado que todo sistema divisionista se contrapone con la escuela inteligente.

Una forma de hacer a la escuela inteligente es a través de su interacción cognitiva con el ambiente, entendiendo que la escuela es un sistema estructurado por unidades de interacciones (Maturana, 1996), que contribuyen a su acoplamiento estructural (Capra, 2000), que supone la autorregulación, organización y la experimentación de cambios en sus estructuras manteniendo su identidad, como todo sistema autónomo, pues el ambiente social genera cambios estructurales, pero no los dirige internamente.

El acoplamiento estructural consiste en establecer diferencias en las interacciones de los sistemas, tanto con el entorno como consigo mismo. En este sentido, un organismo vivo responde a las influencias con cambios estructurales afectando su comportamiento futuro, de manera que un sistema que aprende como la escuela por ejemplo, se acopla en el proceso sin perder de vista su sentido de Ser, este proceso de acoplamiento es considerado inteligente. Para esto, la escuela debe valorar la creación y difusión del conocimiento, y fortalecer los argumentos que ayuden al alumnado a ser feliz, convirtiéndose en una organización con una misión que va más allá de la promesa intelectual.

Para fortalecer la escuela en la construcción de una institución inteligente, se formulan una serie de propuestas que coadyuvan el logro de esta meta, sin menoscabar aquellas que ella misma, a partir de su experiencia, pueda llegar a desarrollar:

1) La formación de estados mentales para la felicidad. La mente es reconocida en las personas y no existe ni funciona sin el cuerpo (Nubiola, 2000), en ella se determinan los estados mentales, donde se integran pensamientos, emociones, sentimientos y espiritualidad, afectándose los sistemas cognoscitivos, el estado de ánimo y las actitudes frente a los problemas cotidianos, en este sentido muchas actividades escolares no ayudan a la creación y sostenimiento de estados mentales para la felicidad, siendo necesario la formación de estos. La formación de los estados mentales pone atención en lo que se piensa y en el cómo se hace, sus condiciones se revelan en las acciones, en los discursos y en la diversidad de lenguajes y formas de comunicación (Aranguren, 2012). En ellos se recogen situaciones humanas condicionadas por percepciones dadas por fuentes externas y desde una suposición fenomenológica son sensibles, emocionales y racionales.

Formar estados mentales supone la interrelación de redes conceptuales, que nutren el pensamiento para comprender y dar respuestas a nuevas situaciones. Su constitución se sostiene en el pensamiento y la emoción, que no necesariamente se deriva de la razón. Todas las acciones humanas se dan con emoción, y es un estado originado por actos externos, ocasionando la alegría, la pena o la tristeza; es temporal, fluye e interviene y se desvanece afectando la realidad, lo sensible, la conciencia y los conceptos dados a la realidad, por lo que su 
manejo y manifestación depende el bienestar cognitivo y físico. Muchas emociones generan movimientos en los estados mentales incidiendo en el talento, la confianza, la voluntad y la inteligencia.

La formación de estados mentales considera la vida del espíritu ya que "la razón es espíritu" (Hegel, 1966, p. 259), siendo su esencia la capacidad para conocer. Es aquí, donde se hace imprescindible la formación y preparación del estudiantado más allá de sus construcciones racionales, por lo que la formación de su pensamiento, emociones y espíritu coloca a la escuela en una situación diferente, haciendo que sus miembros pasen de un estado mental a otro de mayor profundidad; por supuesto, esto contribuye en hacer a la escuela más inteligente.

2) Tomar distancia de los hábitos inadecuados de generaciones pasadas. Es necesario que las nuevas generaciones de jóvenes reflexionen acerca del alcance de las herencias dejadas por las generacionales pasadas, y cómo afectan el progreso evolutivo, tanto a nivel personal como cultural, entendiendo que se vive en un mundo envejecido, y que las generaciones que nacen hoy, crecen y viven en sociedades con hábitos ya sistematizados en sus costumbres y formas de vida.

Las escuelas reproducen los estilos de vida incidiendo en las conductas sociales, y esas reproducciones son los esquemas de las nociones que orientan su formación (Arendt, 1968). Las niñas y niños representan lo nuevo de la sociedad y son formados por estructuras muy antiguas. Es necesario orientar la revelación de aquellos hábitos generacionales que afectan la vida futura del estudiantado y aprender con este a hacer distancia mediante el hábito del entendimiento:

Este hábito filosófico (que no tiene que ver estrictamente con la filosofía en sentido académico) se podría caracterizar como «capacidad de juzgar», tener «buen juicio», incluso, «buen gusto», en un sentido no reductivamente esteticista, este «conocimiento filosófico» es la «perfección o virtud del intelecto». El ejercicio de dicha virtud consiste en la capacidad de «hacerse cargo, de «captar» lo universal en lo particular o, en otras palabras, de arropar un dato particular con la «idea» que le corresponde y da sentido en el conjunto (Torralba, 2013, p. 70).

La enseñanza y práctica del buen juicio es fundamental en la construcción de nociones para la toma de decisiones, que permitan a las nuevas generaciones hacer diferenciaciones y armar nuevos proyectos; en este sentido, Heidegger (1998) señala que las escuelas enfrentan estructuras sociales con falta de distanciación que impiden la toma de decisiones. Siendo imprescindible el desarrollo de estados mentales para la formación del hábito del entendimiento que ayude a enfrentar con libertad, por ejemplo, situaciones de género que repercuten en la formación del estudiantado. En este contexto, las familias han creado estigmas, que en muchas ocasiones son reforzados por las instituciones escolares.

Las nuevas generaciones deberán enfrentar problemas sociales relacionados con la salud, violencia, embarazos no deseados, infecciones de transmisión sexual, VIH y sida. Muchos de estos problemas ocurren porque no hay distancia que permita su comprensión y facilite la toma de decisiones en libertad, ya sea para aceptarlos o rechazarlos. Los sistemas educativos no hacen énfasis en la formación de este hábito (Pérez, 2000), convirtiéndose en un reto educativo, sobre todo, si se plantea la felicidad como paradigma escolar.

Las generaciones venideras deben superar la grave crisis de comprensión que vive el mundo. Los procesos educativos deben hacer una distancia prudencial con el pragmatismo en las aulas y darle importancia a la necesidad que tienen los niños, niñas y adolescentes de comprender el mundo del adulto. Se debe valorar la convivencia entre jóvenes y adultos, porque estos últimos son responsables de la formación de las nuevas generaciones y su aprendizaje representa la herencia, la cual debe ser comprendida para luego ser modificada y las sociedades evolucionen. En este sentido, las aulas de clases son referencias para el estudio de la noción del mundo, del entorno, de los proyectos sociales y del futuro. Es obligación de todas las personas que conforman la escuela desarrollar herramientas para el dominio y socialización del conocimiento, así como su contribución en la reelaboración de los proyectos de vida a partir del ejercicio de distanciación de aquello que hace nociva la relación de la persona con el mundo. Se trata de desarrollar la capacidad de juzgar mediante el 
hábito del entendimiento, el cual deberá permanecer de por vida y "cuyos atributos son la libertad, equidad, serenidad, moderación y sabiduría" (Torralba, 2013, p. 70).

3) La construcción del sentido común. Según Arendt (2002), la sociedad occidental se enfrenta a la pérdida del sentido común representado por la individualización del mundo interno de la persona, haciéndolo incapaz de confiar en otros seres. La formación del sentido común está basada en la relación de lo individual con el conjunto, y favorece las relaciones orientadas hacia el bien común. Este fin busca la consolidación de la responsabilidad social en la persona. Orienta el sentido de la realidad y beneficia el conjunto, facilitando un orden que asegura que cada persona cumpla sus propios fines en libertad y desarrolle su propia personalidad, en conjunto con los otros.

La consolidación del sentido común en la escuela supone un pensamiento común que oriente el desarrollo del bienestar de cada una de las personas que ahí conviven y comparten. La idea es que la escuela fortalezca esquemas de formación que rompan con toda noción individualista. La pérdida de la autoridad y la fractura entre el personal docente y el alumnado, el distanciamiento de la sociedad y la escuela, el abandono cognitivo y el intento de sumergir a la niña y al niño en un mundo envejecido, habla de una pérdida del sentido común, o la destrucción de la noción del mundo que pertenece a todos, o lo que se consideraría la individualización de la sociedad.

4) El desarrollo de las competencias cognitivas mediante rutas de aprendizajes. La escuela se enfrenta a la crisis del abandono cognitivo, siendo uno de los más graves problemas que viven los niños, niñas y adolescentes la incapacidad de comprender y no poseer herramientas intelectuales que les permita leer y entender con propiedad (García, Arévalo y Hernández, 2018).

Las escuelas deben fundamentar sus procesos de planificación y evaluación con base en rutas de aprendizajes (Hernández y Aranguren 2016), una forma de romper con la estricta medición del rendimiento académico y el esfuerzo por cambiar las conductas. Deben construirse espacios sociales creativos para el desarrollo de talentos y estados mentales armónicos, flexibles, en movimiento, y constructores de rutas para aprender. Las rutas de aprendizajes son caminos, métodos, fórmulas que elabora el y la docente para abordar de manera inteligente, la enseñanza en el aula. Permiten que estudiantes pasen de un nivel cognitivo a otro y los ayuda a crear hábitos mentales para aprender. Mediante su aplicación se construyen, organizadamente, procesos de adquisición, procesamiento, ejecución, transferencia y sistematización de la información y del conocimiento. Todo ello, para lograr la comprensión, o sea, entender lo que significa el material trabajado.

Comprender supone la demostración de actividades como la descripción, la comparación, la contrastación y la explicación (Barkley, Cross y Howell, 2007). Ejerce una función importante en el entendimiento de conceptos, recuerdos, y relación con la vida. La comprensión es una función cognitiva que abarca, no solo la información, sino el procesamiento, sistematización y transferencia. Por ello, el desarrollo de las competencias cognitivas mediante rutas de aprendizajes consolida estados mentales para la felicidad y, por supuesto, hace a las escuelas inteligentes

5) El fortalecimiento de las interacciones mediante el aprendizaje cooperativo. El aprendizaje cooperativo es un modelo pedagógico de interacción, su objetivo es hacer que el alumnado trabaje en conjunto para aprender y hacerse responsables de lo que adquieren. La idea es que aprendan como equipo, por ello, la adquisición y transferencia se dan en colectivo, mediante prácticas sostenidas en las recompensas, la responsabilidad individual, la posibilidad del éxito en conjunto, el liderazgo distribuido, los agrupamientos heterogéneos, la interdependencia positiva, la adquisición de habilidades cognitivas y sociales y la autonomía grupal (Ferreiro y Espino, 2009). En su aplicación se desarrollan destrezas sociales que motivan la colaboración y cooperación con el objeto de mejorar la producción intelectual. Se valora la confianza, la comunicación, el apoyo y la capacidad para resolver conflictos. La cooperación contribuye con la formación, la disciplina, el liderazgo y con la capacidad para manejar la diversidad de conflictos suscitados en los equipos, siendo importante la relación de la persona con su entorno, como un medio para fortalecer las capacidades mediante la ayuda mutua. 
6) El desarrollo de las competencias sociales. La escuela debe garantizar el espacio para que el estudiantado desarrolle sus habilidades sociales, son muchas las personas jóvenes que asisten a las escuelas atraídas por las actividades de tipo social, como jugar, compartir, pasear, conversar y hacer amistades ya sea con sus pares o con sus docentes. La socialización supone la interacción, y en las escuelas ellas determinan las relaciones y los modos de convivencia, siendo imprescindible que los jóvenes definan el significado de sus actuaciones y relaciones, ajusten sus acciones y normas y las transgresiones se conviertan en sistemas de aprendizajes. De manera que la escuela es un centro de atracción para la socialización y encuentro entre pares. Aunque las personas mantengan anhelos y deseos de aprender y alcanzar metas académicas, es el espacio social donde crean encuentros en función de sus intereses, nociones y posturas. No solo asisten a clases para responder a los proyectos académicos sino también para convivir (Aranguren, 2017).

La escuela materializa las necesidades de relación de los jóvenes para compartir sus aspiraciones personales, pero puede convertirse en el espacio de distribución de estupefacientes, armas, así como toda la carga cultural aprehendida en las comunidades donde viven. Estas interacciones son el reflejo de la realidad, son el "espejo de lo que ven, palpan y viven, en el resto de ámbitos en los que se desarrollan” (Morollón, 2005, p. 67), por ser la escuela el espacio organizado donde pasan a diario el mayor tiempo de su vida.

7) La formación docente. La formación es un proceso de influencia sistemática en función de un proyecto tanto de vida individual como colectiva. En el caso del personal docente, la idea de la formación es hacer una vinculación entre la teoría y la práctica, por lo que ninguna acción formativa puede tener sentido si los elementos ahí adquiridos no son confrontados en un espacio contextual que los valide, de modo que la escuela es el espacio donde podrían confrontar lo aprendido con la experiencia. Así, la formación se enfrenta de manera sensible, a las influencias sociales con las contradicciones que puedan generarse. Es un proceso de articulación de prácticas, por lo que cumple dos funciones de interés: como práctica de enseñanza y como apropiación del oficio docente (Gudiño, 2014).

Las escuelas necesitan construir organizadamente un proyecto de formación que responda al personal docente y al estudiantado, y asumir un conjunto de condiciones educativas, sociales, económicas, culturales y políticas que recupere la noción de formación de la escuela que facilite la creación del perfil docente y su disponibilidad con las instituciones. Esto implica una visión compartida que precise los aprendizajes y los conocimientos y experiencias. Desde esta perspectiva, el aprendizaje se hace relevante y sometido a un proceso basado en la crítica y el diálogo, donde las dimensiones socioculturales y las relaciones con las dinámicas transformadoras del ambiente y de las estructuras educativas, contribuyen con los cambios y la humanización de la escuela. Bajo este análisis, la formación docente solo puede ser concebida con base en el enfoque de la educación permanente, entendida como la oportunidad que tienen las personas profesionales en educación de participar voluntariamente en la planeación, desarrollo y evaluación de su propia formación (González y Barba, 2014; Lupión y Gallego, 2017).

Es necesario desarrollar, tanto individual como institucionalmente, el significado del valor de la responsabilidad, del respeto y la crítica. Así como experimentar la reflexión de la práctica y sobre la práctica, con el objeto de generar transformaciones en la actividad educativa y en las situaciones y condiciones que limitan el aprendizaje del alumnado.

8) La investigación como práctica de acción y formación. Elliott (1993) señala que la investigación en la escuela genera nuevas concepciones de aprendizaje, de enseñanza y de evaluación, así como originales mecanismos de comunicación con contenidos cooperativos y de ayuda mutua. En este sentido, la investigación debe ser concebida inherente a la acción educativa docente. Cualquiera que sea la orientación de la investigación en educación, esta deberá responder a los problemas y plantear soluciones innovadoras y creativas.

La investigación, la supervisión y la evaluación, dinamizan los procesos de formación docente, enriqueciendo y perfeccionando los procesos educativos, por tal razón la profesión docente no debe considerarse como un todo acabado y cerrado, sino como un proceso de formación continua y permanente. 
De manera, que la investigación en el aula supone para los profesionales de la docencia, estudiar su labor y su repercusión en el alumnado, "en esta situación, el profesor se preocupa por comprender mejor su propia aula" explica Stenhouse, (2007, p. 211). El desarrollo profesional del personal docente depende de su capacidad para investigar su ejercicio. Esto orienta la comprensión de su actuación, convirtiendo la investigación en un elemento formativo de suma importancia, pues permite que el sujeto desarrolle la capacidad de discernimiento y análisis profundo de las situaciones que experimenta en el aula, sensibilizándose en cuanto a su acción y necesidad de considerar las experiencias escolares como aportes a su formación permanente.

En ese sentido, la formación del personal docente en el área de la investigación escolar no puede ser aislada y de manera individualizada. Para que un educador se forme investigador es imprescindible que trabaje al lado de otras personas docentes - investigadoras. El hecho de centrar la formación del personal docente en la investigación convierte al proceso en un elemento rectificador de deficiencias, donde se identifican las partes que funcionan de manera incorrecta y se encuentran los recursos para hacer funcionales los sistemas internos.

La formación centrada en la investigación supone para el personal docente: solucionar problemas dentro y fuera del aula; planificar y evaluar estrategias, ideas y actividades que superen las deficiencias en los procesos de enseñanza y del aprendizaje; sensibilizarse ante los fenómenos que ocurren en el entorno; asumir cambios producto del aprendizaje y de la labor; la oportunidad para reflexionar de los hechos que vive con el alumnado y compañeros de trabajo y asumir la investigación como un instrumento que orienta a cada estudiante hacia la solución de sus problemas. Con la investigación se mejora la calidad de la enseñanza y del currículo; y, dependiendo de la capacidad para adoptar una actitud investigadora, no solo de su propio modo de enseñar sino también de las influencias del entorno, el profesorado puede alcanzar un sentido más crítico de su acción y examinar los conocimientos alcanzados.

Esto sugiere pensar, por ejemplo, que la estructura de elaboración de esquemas de planificación y administración del aula no puede plantearse como se ha venido haciendo, bajo concepciones convencionales y siguiendo estrictamente los currículos oficiales. Debe plantearse la elaboración de proyectos que fortalezcan los procesos de enseñanza, y que estén basados en las necesidades del estudiantado y del personal docente. Se sugieren las herramientas que aportan la Investigación-Acción y la Etnografía, pues a partir de ellas se adquieren aprendizajes valiosos para acciones posteriores. De este modo, el personal docente adquiere las siguientes ventajas: reconoce los problemas y plantea posibles soluciones, genera conocimientos innovadores, integra las competencias que ejerce como docente y modifica sus conductas que no satisfacen el desarrollo de los aprendizajes.

9) La reflexión y sistematización de la práctica. La sistematización se encuentra conectada con la evaluación, planeación, organización y autogestión. Supone ordenar y describir coherente y objetivamente los eventos y conocimientos, de sujetos ubicados en un espacio histórico-contextual y sociopolítico. Permite la organización de la información, la reconstrucción teórica de los eventos, la evaluación de los sistemas y procesos, y propone acciones para el mejoramiento de las experiencias. Su práctica problematiza e identifica los conflictos y contradicciones individuales y grupales dado que es un proceso donde se articula, reflexiona, produce y socializa el desarrollo de las experiencias y de los discursos implícitos en ellas. Cuando se sistematiza se hacen interpretaciones y jerarquizaciones de los hechos y fenómenos; se hacen juicios que deberán ser socializados y valorados para que tenga sentido en la realidad que se sistematiza. En este sentido, se contrastan lo cual es el proyecto inicial y el resultado del proyecto definitivo.

La sistematización se ubica y se interpreta desde dos tareas: teórica y metodológica. La teórica busca la representación de la experiencia desde un nivel abstracto, con el fin de generalizar las interpretaciones que surgen de los eventos o fenómenos que sé vivencian en la realidad. Desde la apreciación metodológica puede funcionar como un sistema de investigación, a partir del método de la reflexión se analiza los discursos y las acciones para descubrir las situaciones que limitan las decisiones y las prácticas efectivas. Esto hace a este proceso consciente de la captura de los significados de la acción y sus consecuencias, donde la organización de las experiencias y de las teorías implícitas en ellas, contextualizan la praxis y facilitan la comunicación del 
conocimiento. Como investigación, la sistematización es un método de análisis que recupera el conocimiento implícito en las experiencias, organiza los conocimientos para ofrecerlo como orientación a otras experiencias similares, generaliza a partir de la información que analiza y comunica y difunde el conocimiento. Por ello, es necesaria en las escuelas, ya sea como medio para develar los discursos implícitos en las prácticas o como forma de reconstrucción y evaluación de las experiencias en las aulas.

10) El trabajo de campo como ejercicio para la enseñanza de la recreación y la espiritualidad. Las escuelas inteligentes buscan espacios naturales para ayudar a su alumnado y profesorado a aprender y desarrollar la enseñanza con una perspectiva práctica; para ello, los Parques Nacionales y recreativos, así como los espacios urbanos son escenarios para llevar a cabo actividades académicas. En ellos, el estudiantado valora y reconoce las potencialidades de los espacios, tanto sociales como culturales, así como aquellos factores climatológicos que ayudan a la comprensión de nociones en ciencias naturales.

Es imprescindible que el estudiantado haga mediciones de variables atmosféricas como temperatura ambiental, humedad relativa del aire, velocidad del viento; variables de biodiversidad y las diferencias de los estratos, descripción de los suelos y estudio organolépticos de estos, descripción de ambientes, reconocimiento y diferenciación de biomas, entre otros aspectos de interés en concordancia con la recreación, compartir y con el desarrollo espiritual que pueda darse en el encuentro de la persona con la naturaleza. La idea es desarrollar habilidades para reconocer variables que definen los biomas y biotopos, consolidar el encuentro fraterno entre los grupos, fortalecer la autoestima y el desarrollo mental y espiritual.

11) Vínculos con agentes externos. La escuela inteligente se esfuerza por acercarse a las instituciones sociales de mayor relevancia cultural, entre ellas la familia, las iglesias, las empresas, las universidades y las organizaciones civiles. Compartir con las familias mediante encuentros, reuniones y celebraciones conduce a la institución a conocer a fondo las diversas realidades de sus estudiantes, sus necesidades y aspiraciones. Facilita la comprensión de las dificultades con respecto a la convivencia. Por lo general, muchas familias requieren ser reconstruidas por razones de índole humana, social, económica y cultural, y necesitan de la escuela para crecer y superar los obstáculos que viven a diario. Por otra parte, las iglesias tienen un papel fundamental en la formación moral de las personas jóvenes, y las escuelas laicas con su diversidad de credos representan una oportunidad de apoyo en la consolidación de redes y equipos de trabajo en función del mejoramiento, no solo en la calidad de la formación, sino también en las relaciones humanas y en la espiritualidad.

En la vinculación con las universidades, ya sea mediante la prestación de servicios comunitarios o pasantías, la escuela crea dinámicas con la participación del estudiantado de manera entusiasta, comprometedora y respetuosa. Estas relaciones pueden llegar a crear y consolidar espacios sociales creativos que permiten la transferencia de información entre las partes involucradas y el manejo adecuado de las relaciones humanas (Aranguren, 2017). Por otra parte, las empresas, sean estas del Estado o privadas, pueden ofrecer oportunidades de enseñanza en actividades que permitirían que el estudiantado aprenda oficios específicos. Esta conexión facilitaría el aprender - hacer en el marco de oficios relacionados con la industria, el comercio y el hogar (Blaslov, 2015).

12) La reconstrucción de las relaciones entre docentes y estudiantado. En la relación docente estudiantado se captan vivencias amenazantes o beneficiosas dependiendo de las circunstancias. Muchas de ellas generan desequilibrios, distanciamientos y rupturas. En este sentido, cada docente está llamado a establecer vínculos para que no se pierda la relación y los afectos, siendo necesario una revisión humilde de su actitud la cual permita las reconstrucciones de la relación.

Un ejemplo de una relación armónica entre estos dos sujetos es tratado con sencillez y madurez en la película El hombre sin rostro de Gibson (1994), donde se relata la historia de un maestro con el rostro desfigurado y refugiado en su casa aislado de todos. Un día recibe la visita de un niño con deseos de prepararse para la presentación de una prueba en una academia militar, esta necesidad facilitó una amistad basada en 
el respeto, la prudencia y admiración, siendo mal vista por la sociedad, originándose presiones para que la relación terminara y el maestro tomara la decisión de salir del pueblo para no regresar jamás.

Aun con todos los prejuicios, es necesario rescatar la noción de amistad entre estos dos sujetos, en el marco de los preceptos de la fraternidad como enseñanza. La fraternidad cobra sentido en la responsabilidad, cuido, respeto y conocimiento que se tiene por los otros (Fromm, 2014), noción con origen en la cultura judía, para ser visto con agrado por Dios y por su relación muy cercana a la noción de la caridad (González, 2007). La fraternidad hace posible la creación de espacios públicos para la convivencia. La enseñanza bajo este precepto hace necesario la promoción de prácticas cooperativas, de respeto y comprensión entre estudiantes, (Slavin, 1999; Johnson, Johnson y Holubec, 1999 y Ovejero, 1990), sin olvidar los encuentros fraternos con el medio ambiente. La fraternidad es base para la excitación o los cambios, y las niñas, niños y adolescentes no tienen herramientas para movilizar por sí mismos sus estados mentales, por ello requieren ser entrenados y ayudados, y esto solo lo puede hacer un cuerpo docente que actúe en condición de amigo.

El estudiantado requiere de aliados para lograr el conocimiento. Castaneda (1986), señala que el aliado beneficia al aprendiz, porque un ser humano de conocimiento necesita de compañeros que le permitan ayudarse en el camino del saber. De manera, que la práctica dentro del aula debe facilitar la alianza armónica entre docentes y estudiantado para construir oportunidades de aprendizajes de mayor alcance.

13) La valoración del trabajo. Las escuelas inteligentes ofrecen formación para el trabajo, así como oportunidades para que la persona joven trabaje y aprenda un oficio. Para ello, es necesario la creación de talleres de Educación para el Trabajo en diversas áreas de oficios. El estudiantado que allí asista deberá ayudarse mutuamente y cooperar entre ellos "a fin de que no haya ningún compañero que se quede sin aprender verdaderamente los oficios fundamentales" (Blaslov, 2015, p. 92). Los procesos internos en las aulas deben crear en las personas menores de edad el orgullo de trabajar en las tareas escolares. Tanto la fraternidad, la cooperación y las nociones de justicia pueden ser creadas mediante el trabajo participativo y colaborativo.

14) El fortalecimiento de la espiritualidad. Las escuelas inteligentes educan desde adentro, o sea desde la interioridad. Este ejercicio educativo busca mejorar y fortalecer las nociones de los derechos humanos en las escuelas y en todos los ámbitos de la sociedad. El fortalecimiento de la espiritualidad no supone el trabajo de catequesis o de adoctrinamiento religioso, va más allá de este sentido. Es comprender que todos los seres humanos poseen espíritu y tiene necesidad de experimentar una vida fraterna con sus semejantes. Esta perspectiva de la formación responde a la categorización de la inteligencia dentro del campo de lo espiritual, haciendo de esto una competencia de gran alcance humano que permitiría darle sentido a todos los saberes que surgen y se manejan en las escuelas (Azofra, 2018). En este sentido, el desarrollo de la interioridad supone con ello la promoción, práctica y estudio de todo aquello relacionado con la creación artística, como lo son la literatura, escultura, arquitectura, pintura, música, danza y cine, ya que con estas disciplinas la persona puede manifestar el alcance de su estado mental, además de ser un medio conducente a la trascendencia.

15) La profundización en la educación emocional. La educación de las emociones y la Inteligencia Emocional es una tarea necesaria en la educación, es imprescindible considerar estas habilidades para el desarrollo evolutivo y socioemocional de los niños, niñas y jóvenes. La educación emocional tiene como finalidad desarrollar competencias emocionales que favorezcan el bienestar personal y social, para ello requiere la formulación de programas fundamentados en propuestas reconocidas por académicos en el área, profesores debidamente formados y animados a los cambios curriculares, materiales elaborados y orientados por recomendaciones de teóricos reconocidos, así como una evaluación y revisión permanente de los programas

Bisquerra (2000) define la Educación Emocional como un proceso educativo continuo y permanente, que potencializa el desarrollo emocional y los procesos cognitivos. Esta práctica brinda competencias para el manejo de las emociones para que la persona afronte los retos de la cotidianidad; su objetivo es aumentar el bienestar y por ello debe ser intencional y sistemática. Estos procesos incluyen el autoconocimiento, el autocontrol, la empatía, la escucha, la resolución de conflictos y la cooperatividad; debe estar dirigida con 
base al desarrollo de la capacidad para comprender emociones, para expresarlas de forma productiva y para escuchar al otro, sin menoscabar la importancia de ser empático en las relaciones con los pares. Para la puesta en práctica de programas de este tipo es fundamental que se formulen estrategias que integren las diversas áreas académicas, a lo largo de todos los cursos y de manera transversal, siendo importante el papel del cuerpo docente, pues por imitación el alumnado aprende a desarrollar su propia Inteligencia Emocional.

16) La promoción y uso de las Tecnología de la Información y de la comunicación. Las escuelas inteligentes no sienten temor de las tecnologías, porque saben que estas herramientas aportan contenidos motivacionales que facilitan la autodidactica y la socialización (Blázquez, Orcos, Mainz y Sáez, 2018). Potencializan la atención del estudiantado haciéndolos independientes. El personal docente está en el deber de orientar su uso de forma creativa, sabiendo que le son útiles para desarrollar procesos de aprendizaje y lograr competencias. Las TIC contribuyen al desarrollo del trabajo en equipo para solucionar problemas, y permite que el estudiantado interactúe, se autoevalúe y reciba feedback simultáneamente. El personal docente puede enriquecer sus actividades de aula, así como fortalecer los contenidos (Miratía, 2011).

17) La democratización del poder escolar. El poder, culturalmente, está asociado con la violencia, el dominio del otro y el establecimiento disciplinar, así como con las restricciones, negociaciones y los modos y contenidos para la toma de decisiones (Batallán, 2003). Está ligado con las jerarquías, las cuales gestionan los procesos internos en las instituciones. En lo que corresponde a la escuela, la administración escolar es una actividad burocrática que responde a protocolos cargados de mandatos y normas, suponiendo que aquellos, que jerárquicamente dirigen la escuela, están obligados ética y moralmente en hacer que dichas reglas se cumplan. Una nueva mirada de la forma cómo se desplaza y se conjuga el poder en las escuelas, considera a la política como medio de resolución de problemas. En tal sentido, se revela una construcción civilizatoria de las nociones de la distribución del poder. Una revisión, profundización y cambios en los modos del manejo del poder y autoridad en las escuelas facilitaría las nuevas reglas para tratar la disciplina y la confianza.

La democratización del poder es necesaria y supone para el estudiantado y el personal docente el riesgo a cambios sustanciales en el seno de las instituciones de gobierno interno. Esto conecta a la escuela con el país y con sus nociones de convivencia y fraternidad. La escuela es el espacio donde las relaciones sociales se reproducen (Ugarte, Alva, Gómez y Gonzales, 2006, p. 16), por lo que la democratización escolar pasa por entender este mismo proceso en la sociedad. Ahora bien, un sistema democratizador de la escuela considera a todas las personas que actúan en ella, así como los espacios de poder, tanto de las y los docentes como del estudiantado.

Cuando se habla de democratizar el poder escolar se supone que ningún cargo de autoridad debe ser eterno, de manera que estos deben pasar por revisiones a través de consultas libres y secretas que faciliten la participación de todas las personas involucradas de esa comunidad educativa. Para ello, las instituciones deben crear mecanismos, protocolos y normas reguladores de los ejercicios internos de participación.

\section{LA ESCUELA INTELIGENTE Y EL CULTIVO DE LA FELICIDAD}

Lo más humano es ser feliz, aun en situaciones inhumanas. Desde esta percepción, la felicidad se caracteriza porque la vida está en condiciones de ejercitar la dicha, por el simple hecho de estar vivo y por ser la vida el bien supremo; cabe preguntarse si las aulas de clases son espacios para promover la felicidad, en ellas diversos grupos de personas conviven por largo período de tiempo, si las escuelas no cumpliesen este papel entonces cabría imaginarse aulas deshumanizadas y en desequilibrio. Perkins (1999) plantea que la escuela inteligente es capaz de diluir la dicotomía sobre qué es lo más importante: el método o los contenidos (el cómo se enseña y lo que se elige enseñar). En educación existe una variedad de métodos muy buenos, pero pierden su eficacia en el proceso de su aplicación debido a que los resultados no responden a los esperados. Lozano (2004) argumenta que esta escuela se revela en la orientación al perfeccionamiento de la persona, a su autorrealización y a la 
relación con la naturaleza, la sociedad y la cultura, por ello se conecta con la formación y enseñanza de la felicidad.

La escuela tiene un compromiso social, que no solo abarca el hecho de ser un mediador político entre el estudiantado y la sociedad con sus representaciones culturales, es necesario establecer la responsabilidad de desarrollar la inteligencia como un bien, tan importante como la vida, por ello, es imprescindible que la persona posea herramientas para abordar las formas y modos de cómo se desarrolla y evolucionan los procesos culturales y humanos donde se desenvuelve. Es necesario cultivar y preparar al estudiantado para la comprensión, suministrándole información y mostrándole los procesos mentales que intervienen en la solución de sus problemas, considerando las tareas, el desarrollo de talentos, sus aprendizajes y reflexión, las estrategias para abordar sus problemas y las experiencias cotidianas, de manera que identifique las oportunidades que el ambiente social y humano ofrece, para que se oriente en el establecimiento de un estado mental que le ayude a vivir en medio de situaciones inesperadas.

Hablar de una escuela inteligente es asumir un currículo que aborde al educando como una persona provista de elementos sensitivos y receptivos los cuales nutren el proceso de desarrollo y evolución del pensamiento, a fin de contribuir con varias razones de vida: la calidad de la existencia, la libertad y la felicidad como resultados del desarrollo de las capacidades, sabiendo que el mundo que se visualiza en el futuro podrá ser comprendido por aquellas personas que estén informadas y sepan cómo procesar la información.

La información y su procesamiento son claves de poder, pero también de la hegemonía de unos pueblos sobre otros mediante los sistemas de libertad y democracia o de la tiranía y esclavitud. La mayor libertad lograda por los seres humanos se sostiene en la noción que desarrollan acerca de la existencia y la acción (Arendt, 1993), dado que la persona solo podrá ser libre cuando posea conciencia del alcance de sus capacidades o talentos (Aranguren, 2012); por ello, es un requisito para las sociedades de hoy día y las del futuro actuar en función de profundizar la democratización de la inteligencia (Machado, 2019) como un valor sociocultural de importancia.

Un proyecto de escuela inteligente encamina sus esperanzas al desarrollo de un modelo educativo que considera el pensamiento como la fuerza potencial para hacer evolucionar los talentos, y con estos el conocimiento. En este caso, es importante señalar que el conocimiento, objeto de aprendizajes en las aulas de clases, representa, de manera seleccionada y sistematizada, los saberes que, culturalmente son de importancia para la sociedad; cualquier reacomodo dado entre la escuela y la sociedad en cuanto al conocimiento y la práctica, siempre va a ser discutido por la clase social y política que la sostiene, hasta dar su consentimiento tácito o expresar libremente su rechazo definitivo.

La evolución del pensamiento, de los talentos y del conocimiento se proyecta, tarde o temprano, en los actos humanos y culturales, queriéndose decir con esto, que los procesos culturales responden a las formas y modos en que se organiza el pensamiento, y con ello los actos. Es necesario aprovechar las aulas de clases para compartir conocimientos y establecer metas comunes, las cuales conduzcan a profundizar el bienestar humano. Si estas no asumen a la persona como el principio y fin de su institución, entonces, se está hablando de un aula deshumanizadora, que no responde con las necesidades sociales de bienestar, felicidad y mejoramiento de la calidad de vida.

Cabe preguntarse qué ocurre con aquellas personas que no han pasado por un aula de clase, o han experimentado algún fracaso escolar, si su experiencia ha influido en el logro o no de su bienestar y en el establecimiento de un estado mental disponible para superar las situaciones adversas y disfrutar de las condiciones favorables.

Las situaciones que desequilibran el sistema educativo lo hacen vulnerable a circunstancias adversas, no siendo sencillo abordar la formación, mucho menos pensar en la integración y equilibrio cuando la política queda sujeta al mantenimiento del sistema. En este caso, cabe destacar lo señalado por Herrera (2004), cuando indica que los factores más importantes en los que el sistema educativo se ha establecido son reducir 
el fracaso escolar, el bajo rendimiento académico, la deserción, la repitencia, la necesidad de comprensión de textos, entre otros, que solo han originado desequilibrio y desarmonía en las aulas de clases.

La consecución de elementos que ayuden a entender la felicidad pasa por el requisito de que se debe partir de la realidad de cada cual, comprendiéndose esto como el desarrollo de la capacidad de conocerse a sí mismo, de manera que, mediante la práctica de la introspección y la escucha del otro, la persona puede superar la ignorancia de sí. La práctica del conocimiento de sí mismo resulta ser un arte, que implica "el conocimiento del cuerpo, de los sentidos, los sentimientos, los pensamientos propios" (Eckhart, en Huxley, 1999, p. 216), es, básicamente, la representación de la inquietud de sí mismo planteado por Foucault (2002), siendo la ignorancia de sí mismo la vía que conduce a una visión irreal de la persona. Plantearse el conocimiento de sí mismo está suficientemente argumentado por Schopenhauer (2004), cuando señala lo siguiente:

Lo que uno es contribuye más a la felicidad que lo que uno tiene o lo que uno representa. Lo principal es siempre lo que un hombre es, por consiguiente, lo que posee en sí mismo; porque su individualidad le acompaña en todo tiempo y en todo lugar y tiñe con su matiz todos los acontecimientos de su vida. Es toda cosa y en toda ocasión, lo que le afecta primero es él mismo. Esto es cierto aun para los goces materiales, y, con mayor razón, para los espirituales (p. 12).

Revelándose, al menos, dos consideraciones de importancia, la primera que la felicidad pasa por conocerse a sí mismo y, la segunda, que este conocimiento como expresión humana se alcanza en la relación con los otros, donde la persona se manifiesta frente a los demás desde su individualidad como expresión única.

La preparación de los niños y niñas para actuar frente a la realidad con la cual se enfrentan, en otro sentido, darle herramientas para que vivan y disfruten de lo que experimentan, es hablar de una escuela para la felicidad planteada desde una pedagogía desde lo erótico, el goce, la ternura, lo sublime, donde se integren lo corporal (los sentidos), lo etéreo (el alma) y lo intelectual (la razón), es plantearse la necesidad de generar un proyecto educativo basado en el conocimiento de sí mismo, y hacer de este proceso un verdadero arte, o sea, una combinación amplia de una serie de situaciones humanas que contribuyan a la generación de la armonía.

Otro aspecto por considerar es el hecho de que, al hablar de una escuela inteligente, se aborda de manera sutil otro tipo de escuela que no se estima dentro del contexto y valor de la inteligencia. Siendo así, la escuela no-inteligente, es aquella que difícilmente promueve el desarrollo del pensamiento y la felicidad. Ella es la escuela en la cual no es calculable su dimensión y su capacidad de aprehensión, pero sí es factible saber acerca de sus resultados. Una escuela lejos de ser inteligente mantiene y sostiene la cultura y todos los efectos humanos, buenos y malos, estableciendo los códigos y símbolos que han podido aprender para dosificar sus experiencias.

Es importante comprender el aula de clases en el contexto de lo que significa la integración, entendiendo que ella representa un espacio social donde todos sus integrantes se enfrentan con sus paradigmas, a partir de sus fortalezas y debilidades, y hacen de la práctica cotidiana un frecuente debate acerca de los métodos, problemas y normas que los rigen.

\section{LA FELICIDAD Y EL DESAFÍO DE LA TÉCNICA}

El concepto de escuela se sostiene también en preceptos utópicos o esperanzadores en la noción del proyecto general de sociedad. Cualquier proceso de formación y enseñanza pasa por consideraciones axiológicas del Estado, siendo un ejemplo de ello, las Bases y Principios de las Escuelas Bolivarianas en Venezuela, donde se señala:

Nos proponemos, desde la educación, reconstruir el país, mirar hacia atrás y a hacia adelante, recuperarnos y construir futuro y convivencia. El país no está hecho ni siempre es igual a sí mismo, la patria hay que hacerla para que esté viva, la patria vive en la medida en que construimos nación desde nuestras diferencias y desde nuestros puntos comunes (Ministerio del Poder Popular para la Educación [MPPE], 2004, p. 11). 
Esta postura se enfrenta a una sociedad en busca de oportunidades para definir la orientación de los procesos educativos, de este modo, la Sociedad Civil Organizada, hacen de la organización una escuela que canaliza y produce conocimientos y experiencias que reorientan los fundamentos que sustentan la educación, generándose una dicotomía Estado - Sociedad sujeta a las nociones sobre cómo debe concebirse el futuro de la educación. Cada una de las partes mantiene criterios que señalan el paradigma de sociedad que se desea, apoyados en intereses, discursos y acciones. En este sentido, cada una de las partes intenta comprender el futuro, que, prácticamente, es noción de posibilidades, de incitación, esperanza y amenazas, es "un invento de última hora que acompañó a la industrialización acelerada, los progresos de la aeronáutica, las nuevas máquinas de guerra y la quiebra generalizada del viejo mundo mítico - religioso tradicional" (Savater, 2001, p. 126).

Las utopías han surgido desde la frustración y de premisas de situaciones no concluidas, convirtiéndose en la demostración de la imposibilidad del poder político en resolver los asuntos humanos. Las construcciones educativas utópicas obligan e imponen los mecanismos de formación y preparación social. La Constitución de la República Bolivariana de Venezuela del año 1999, establece que la educación es obligatoria, y que el Estado está en el deber de asumirlo, "como función indeclinable y de máximo interés en todos sus niveles y modalidades" (Art. 102), pues la exigencia está dada, de modo exclusivo, a la ciudadanía, pero no al Estado.

El acto educativo desde la obligación, como requisito para acceder al futuro deseado, conduce a la necesidad de la represión como fórmula para no perder el futuro. Los actos educativos basados en la crueldad y la represión son una demostración de que el sujeto reprimido no mantiene los requerimientos básicos para acceder al concepto de futuro de quien reprime.

La estructura racional técnica que supone la comprobación empírica de las teorías, por lo que el conocimiento en este sentido, es objetivo y se describe en forma neutral, (Giroux, 1999 y Carr y Kemmis, 1988) y, con ella, la técnica como regla práctica orientada a la aplicación o ejecución de una actividad manual o intelectual, han sostenido la creencia del desarrollo y la redención humana, pretendiéndose señalar que la felicidad absoluta viene dada por la posesión, el control y la certeza técnica de las cosas en el presente, siendo la formación y preparación la fórmula para alcanzar estas metas.

En el intento de comprender la escuela como medio para el ascenso social, Bravo (2003), señala que "prácticamente todos los documentos oficiales asociados a la cuestión educativa, conciben a la escuela como mecanismo de ascenso social y como forma de redención personal; más educación escolar significa más y mejores oportunidades y mayores posibilidades de realización personal” (p. 14), sobre todo si las oportunidades están orientadas al área tecnológica y científica.

Es preciso recordar que la técnica y su conocimiento forman parte de lo que significa la instrucción básica, su enseñanza permite su comprensión y valoración con el fin de hacer un mejor uso de ella, ya que

la ignorancia de los métodos técnicos coloca al individuo cada vez más a merced de otra persona en la vida cotidiana, reduce sus posibilidades de empleo y acrecienta el peligro de que los posibles efectos perjudiciales de la aplicación excesiva de la tecnología - alineación de los individuos, contaminación, etc., acaben por predominar. La mayoría de la gente se beneficia pasivamente de la tecnología o se resigna a ella sin entender nada; por tanto no pueden actuar de ninguna forma sobre ella (Faure, 1980, p. 128).

Como artífice de las bondades tecnológicas de los últimos tiempos, la técnica ha contribuido con la desesperanza en el mundo generándose una crisis ética en lo referente al uso de las tecnologías, discusión sobre la cual evoluciona la historia contemporánea basándose en la "interminable discusión sobre si el hombre debe ajustarse a la máquina o las máquinas a la naturaleza del hombre” (Arendt, 1993, p. 166). La tecnología es la representación del talento humano, por ser la concreción de los conceptos, tanto de la ciencia como de toda la capacidad abstractiva del hombre; es el resultado del pensamiento profundo y abstracto de la humanidad. Este sistema tecnología - técnica, es el signo práctico que satisface, con las herramientas producidas, el saber hacer, pero no conduce necesariamente al sentido de la existencia, pues esta viene dada por el acto de la revelación 
de sí mismo, convirtiéndose el revelar en un serio problema para la humanidad, ya que resulta pertinente que el hombre sepa quién es, más no qué es.

Foucault (2002) considera la técnica como una serie de prácticas o un tanto de ejercicios, que contribuyen con la construcción de la noción del presente, obviándose el concepto de la espera y de la pasión por el devenir. De este modo, el concepto de futuro se mezcla con lo presente mediante ritos, y son estos los que advierten la presunción del futuro. En esto hay que considerar que, definitivamente, la técnica y con ello las nociones progresistas de la modernidad no hacen y no han hecho feliz a las sociedades, para ello es requisito el uso de la inteligencia, no como resultado de la técnica, si no como la experiencia comprensiva de las capacidades y oportunidades del desarrollo humano.

La escuela inteligente surge como propuesta que busca no solo romper con el paradigma tecnocrático, sino también con los obstáculos que impiden comprender las oportunidades del presente como proyección y construcción de las nociones de futuro. De manera que la felicidad, entendida mediante la apreciación del estado mental, no admite condiciones utópicas, más bien se sostiene en todo aquello que procura abordar el presente con todas sus limitaciones y fortalezas a fin de crear, en medio de las posibilidades, escenarios creativos del futuro.

En todo esto de las utópicas posibilidades, la felicidad surge como una idealización imaginaria del futuro y no como proyecto de organización humana logrado mediante el desarrollo de los estados mentales. Aquí es donde la escuela, lejos de crear miradas inciertas del futuro, debe contribuir en la consolidación de ambientes sociales creativos (Parra, 2006) que faciliten la formación para el bienestar personal y colectivo.

\section{Epílogo}

La escuela es el centro social para el desarrollo del aprendizaje y la enseñanza, uno de sus fines es que sus integrantes aprendan a ser felices. Debe estar diseñada para que sus miembros tengan claro que sus procesos internos están dados para que cada persona construya espacios humanos facilitadores de la dicha; este modo de actuar de la escuela la hace una institución inteligente y, con base en esta premisa, se deben formar los estados mentales de las personas.

Una escuela inteligente y comprometida con el bienestar del estudiantado, lo acompaña en los procesos de adquisición y descubrimiento de sus capacidades, y se mantiene comprometida para despertar la creatividad. Es una escuela para el saber hacer. Siendo necesario una serie de elementos académicos que permitan el cultivo del hacer, de manera que desde ahí se alimenten los sentidos y la comprensión de lo que se sabe. Otro aspecto importante es el que sus acciones están orientadas al perfeccionamiento integral e intencional de la persona, a su autorrealización y relación con la naturaleza, la sociedad y la cultura.

La escuela inteligente y promotora de felicidad, supone la apertura a la evolución, investigación y descubrimiento de talentos o capacidades, ya sean estas aprendidas desde la sensibilidad o desde la cotidianidad; por otro lado, debe esforzarse por enseñar hábitos mentales que permitan la resolución de problemas de toda índole. El desarrollo de talentos es, prácticamente, obligatorio, algunas personas lo harán mejor que otras, su ejercicio cotidiano contribuye en su perfección hasta hacerse parte de la vida.

Un aspecto de importancia en la construcción de la Escuela Inteligente es el acercamiento a las familias, pues compartir con ellas mediante actividades debidamente planificadas, permite el conocimiento más profundo de la realidad del estudiantado, sus necesidades y aspiraciones; de igual modo, se facilita la comprensión de las dificultades y formas de convivencia familiar, puesto que muchas familias requieren de la escuela para crecer y superar los obstáculos de cada día.

Este orden de lineamientos estratégicos de alcance educativo busca facilitar la comprensión del aula de clases en el marco de la escuela inteligente, a fin de propiciar la creación de una noción de felicidad como aspiración vivencial-humana de todas aquellas personas que la constituyen. En este sentido, se propone que en el proceso de la enseñanza se profundicen actividades básicas, que contribuyan a que el estudiantado: 
(a) aprenda a leer y a escribir en la búsqueda de la posible perfección en el tiempo; (b) comprenda lo mejor posible los asuntos académicos y todo aquello que le atañe en relación con la vida; (c) valore sus potencialidades o talentos y (d) haga uso de los medios académicos para experimentar y desarrollar la voluntad, la responsabilidad, la contemplación, la inquietud y el conocimiento de sí mismo, la imaginación y la ternura.

\section{REFERENCIAS Bibliográficas}

Aranguren, G. (2012). El favor de los dioses. Caracas: Ediciones de la Memoria Educativa Venezolana.

Aranguren, G. (2017). La escuela como opción y espacio: un análisis desde las concepciones y creencias. Revista Areté, Revista Digital del Doctorado en Educación de la Universidad Central de Venezuela. 3(5). 83 -111. Recuperado de http://saber.ucv.ve/ojs/index.php/rev_arete/article/view/12834

Arendt, H. (1968). Entre el pasado y el futuro. Ocho ejercicios sobre la reflexión política. Barcelona, España: Editorial Península.

Arendt, H. (1993). La condición humana. Barcelona: Paidós.

Arendt, H. (2002). La vida del espiritu. Barcelona: Paidós.

Azofra, E. (Octubre, 2018). Educación en la interioridad. En: T. Carmona. (Presidencia). Gestionar hoy la escuela del futuro. Ponencia presentada en el VI Congreso Pedagógico - Pastoral de la Asociación Venezolana de Educación Católica (AVEC). Caracas, Venezuela.

Barkley, F.; Cross, P. y Howell, C. (2007). Técnicas de aprendizaje colaborativo. Manual para el profesorado universitario. Madrid: Morata.

Batallán, G. (2003). El poder y la autoridad en la escuela. La conflictividad de las relaciones escolares desde la perspectiva de los docentes de infancia. Revista Mexicana de Investigación Educativa 8, (19) 679-704. Recuperado de http s://www.redalyc.org/articulo.oa?id=14001906

Bisquerra, R. (2000). Educación emocional y bienestar. Barcelona: Praxis.

Bisquerra, R. y Hernández, S. (2017). Psicología positiva, Educación Emocional y el Programa de Aulas Felices. Papeles del Psicólogo, 38(1), 58 - 61.

Blaslov, E. (2015). Educación y evangelio. Educación para el trabajo: la experiencia de la iglesia para Venezuela. Caracas: IUPMRAB - UCAB.

Blázquez, T.; Orcos, L.; Mainz. J. y Sáez, D. (2018). Propuesta metodológica para la mejora del aprendizaje de los alumnos a través de la utilización de las impresoras 3D como recurso educativo en el aprendizaje basado en proyectos. Psicología, Conocimiento y Sociedad 8(1) 162 - 193. Recuperado de http://revistas.sena.edu.co/inde x.php/rnova/article/view/1538/1706

Bravo, L. (2003). Educación escolar en Venezuela: claves teóricas, matrícula y población. Caracas: Decanato de Postgrado. Universidad Nacional Experimental "Simón Rodríguez".

Capra, F. (2000). La trama de la vida: Una nueva perspectiva de los sistemas vivos. Barcelona: Anagrama.

Carr, W y Kemmis, S. (1988). Teoria Crítica de la Enseñanza: La Investigación-Acción en la Formación del Profesorado. Barcelona: Martínez Roca.

Carro, A., y Lima, J. (2017). El uso de la web en jóvenes de Educación Secundaria. El caso de un centro escolar de la periferia urbana en el Estado de Tlaxcala, México. Revista Pedagogía, 38(103), 82-106. Recuperado de http:// saber.ucv.ve/ojs/index.php/rev_ped/article/view/14928

Castaneda, C. (1986). Las enseñanzas de Don Juan. México: Fondo Cultural Económica.

Constitución de la República Bolivariana de Venezuela (30 de diciembre de 1999). Gaceta Oficial de la República Bolivariana de Venezuela, 36.860.

Elliott, J. (1993). Cambio Educativo desde la Investigación - Acción. Madrid: Morata.

Faure, E. (1980). Aprender a Ser. La educación del futuro. Madrid: Alianza Editorial. 
Ferreiro, R. y Espino M. (2009). El ABC del aprendizaje cooperativo: Trabajo en equipopara enseñary aprender. México: Trillas.

Fernández, L. (2001). El análisis de lo institucional en la escuela. Un aporte a la formación autogestionaria para el uso de los enfoques institucionales. Notas teóricas. Buenos Aires: Paidós.

Fernández, M. (2013). La inteligencia emocional. Revista de Claseshistoria. (377), 2 - 12. Recuperado de http://ww w.claseshistoria.com/revista/index.html

Foucault, M. (2002). La hermenéutica del sujeto. Curso en el Collège de France (1981 - 1982). Buenos Aires: Fondo de Cultura Económica de Argentina, S. A.

Fromm, E. (2014). El arte de amar. Madrid: Paidós.

García, J. (2016). La introducción de la escala de Stanford - Binet en el Paraguay. Revista Interacciones, 2(1), 65-83. Recuperado de https://dialnet.unirioja.es/servlet/articulo?codigo $=5759440$

García M.; Arévalo M. y Hernández C. (2018). La comprensión lectora y el rendimiento escolar. Cuadernos de Lingüistica Hispánica (32), 155-174.

Gibson, M. (Productor) (1994). El hombre sin rostro. [Película]. Warner.

Giroux, H. (1999). Teoría y resistencia en educación. México: Siglo Veintiuno Editores.

González, G. y Barba, J. (2014). Formación permanente y desarrollo de la identidad del profesorado desde la perspectiva grupal e individual. Revista de Curriculum y Formación de Profesorado, 18 (1), 397-412. Recuperado de http:// www.redalyc.org/pdf/567/56730662023.pdf

González, E. (2007, Abril 29). Jesús, según el Papa. El Pais. Recuperado de http://www.elpais.com/?d_date=20070 429

Gudiño, A. (2014). Formación docente, enseñanza y didáctica. Una Investigación 6(11), 93-113. Recuperado de http ://biblo.una.edu.ve/ojs/index.php/UNAINV/article/view/1339/0

Heidegger, M. (1998). El ser y el tiempo. Bogotá: Fondo de Cultura Económica.

Hernández, Y. y Aranguren, G. (2016). Patrón tecnopedagógico: Ruta de aprendizaje basado en actividades comprensivas. Revista Vinculos, 13(2), 30 -39.

Hegel, G. (1966). Fenomenología del Espiritu. México: Fondo de Cultura Económica.

Herrera, M. (Mayo, 2004). Educación y superación de la pobreza en Venezuela. En: Parra, G. (Director). Globalización, Ciudadanía y Educación. Ponencia presentada en el I Seminario Nacional de la Línea de Investigación Dinámicas Psicosociales y Ambientes de Aprendizaje. Universidad Nacional Experimental “Simón Rodríguez”, Decanato de Postgrado. Caracas, Venezuela.

Huxley, A. (1999). La filosofía perenne. Buenos Aires: Sudamericana.

Johnson, D. Johnson, R. y Holubec. E. (1999). El aprendizaje cooperativo en el aula. Buenos Aires: Paidós.

Lozano, A. (2004). El aula inteligente: ¿hacia un nuevo paradigma educativo? Revista Electrónica de Investigación Educativa, 6(2). Recuperado de http://www.scielo.org.mx/pdf/redie/v6n2/v6n2a8.pdf

Lupión C. y Gallego, M. (2017). Compartiendo la mirada: una experiencia en práctica reflexiva para formación permanente. Revista Electrónica Interuniversitaria de Formación del Profesorado, 20(1), 127-44. Recuperado de https://dialnet.unirioja.es/servlet/articulo?codigo $=5969913$

Machado, M. L. A. (2019). La democratización de la inteligencia. Recuerdos de un futuro. Caracas, Venezuela: Edición digital. Recuperado de https://larevoluciondelainteligencia.jimdofree.com/libros-de-lam/

Maturana, H. (1996). La realidad: ¿Objetiva o construida? Fundamentos biológicos del conocimiento. Guadalajara: Anthropos Editorial.

Ministerio del Poder Popular para la Educación. (2004). Escuelas Bolivarianas. Avances Cualitativos del Proyecto. Recuperado https://www.oei.es/historico/quipu/venezuela/Escuelas_Bolivarianas.pdf

Miratía, O. (2011). Diseño Instruccional Para el Desarrollo de Contenidos Educativos (DI-DCE). Recuperado de https ://es.slideshare.net/omiratia/diseo-instruccional-para-el-desarrollo-de-contenidos-educativos-didce 
Morollón, P. (2005). La diversidad cultural y sus conflictos, un camino hacia la interculturalidad. En: Santos Guerra. M.A. (Coord.). Aprender a convivir en la escuela. (pp. 67-88). Sevilla: Akal -S.A.

Nubiola, J. (2000). Perspectivas actuales en la filosofía de lo mental. Espiritu, 49, 13-24. Recuperado de http://www .unav.es/users/Articulo45.html

Ovejero, B. (1990). El aprendizaje cooperativo: una alternativa eficaz a la enseñanza tradicional. Barcelona: Promociones y publicaciones universitarias S.A.

Parra, G. (2006). La Universidad se Reforma. Caracas: Metrópolis. C. A.

Pérez, D. V. (2000). La educación liberal como la formación del hábito de la distancia. ASP Research Paper, 37, 609 626. Recuperado de https://www.researchgate.net/publication/268177765_LA_EDUCACION_LIBERAL_ COMO_LA_FORMACION_DEL_HABITO_DE_LA_DISTANCIA

Perkins, D. (1999). La escuela inteligente. Barcelona: Gedisa.

Prieto, E. (2018). La psicologización de la educación: implicaciones pedagógicas de la Inteligencia emocional y la psicología positiva. Revista Educación XXI, 1(21), 303 - 320. Recuperado de http://www.redalyc.org/articulo .oa?id=70653466014

Savater, F. (2001). Sobre Vivir. Barcelona: Ariel.

Slavin, R. (1999). Aprendizaje Cooperativo. Teoria, Investigación y práctica. Sao Paulo: Aique Grupo Editor S.A.

Schopenhauer, A. (2004). La Sabiduría de la Vida. En torno a la filosofía. El Amor, Las Mujeres, La Muerte y Otros Temas. México: Porrúa.

Stenhouse, L. (2007). Investigación y desarrollo del curriculum. Madrid: Morata.

Torralba, J. (2013). La idea de educación liberal. De cómo se inventaron las humanidades. En: J. Arana (Ed.). Falsos saberes. La suplantación del conocimiento en la cultura contemporánea (pp. 61 - 74). Madrid, España: Biblioteca nueva.

Ugarte, D., Alva, J., Gómez, C. y Gonzales, E. (2006). Aprendiendo a democratizar la escuela pública. Una experiencia en independencia de Lima Metropolitana. Recuperado de http://tarea.org.pe/images/Ugarte_Sistematizacion_ 20070601.pdf

\section{BY-NC-ND}

